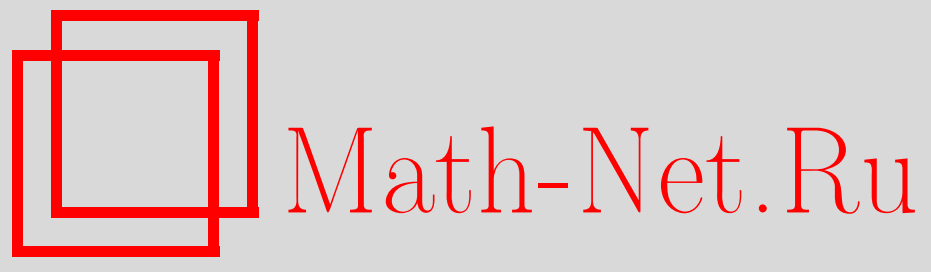

Э. А. Аринштейн, Нелокальная теория поверхностного натяжения простой жидкости, ТМФ, 2006, том 148, номер 2, 323-336

DOI: https://doi.org/10.4213/tmf2089

Использование Общероссийского математического портала Math-Net.Ru подразумевает, что вы прочитали и согласны с пользовательским соглашением http://www . mathnet.ru/rus/agreement

Параметры загрузки:

IP : 3.80 .181 .102

26 апреля 2023 г., 18:10:16

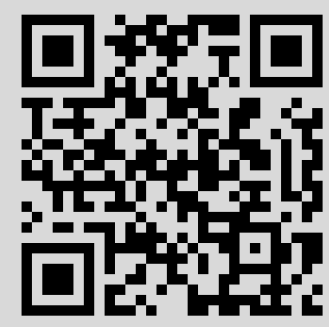




\title{
НЕЛОКАЛЬНАЯ ТЕОРИЯ ПОВЕРХНОСТНОГО НАТЯЖЕНИЯ ПРОСТОЙ ЖИДКОСТИ
}

\begin{abstract}
Рассмотрен метод выделения из общего выражения для термодинамического потенциала двухфазной системы жидкость-пар поверхностного вклада, имеющего вид нелокального функционала от структурной функции переходного слоя. Отмечена возможность использования связи между термодинамическими потенциалами равновесных объемных и поверхностной фаз для модификации полученного разложения. Получен аналог первого интеграла Эйлера вариационной задачи для функционала поверхностной энергии. Приведены оценки параметров, характеризующих поверхностный слой и его асимптотику.
\end{abstract}

Ключевые слова: фазовое равновесие, поверхностное натяжение, структурная функция, корреляционные функции, термодинамический потенциал.

\section{1. ВВЕДЕНИЕ}

Трудность построения последовательной теории жидкого состояния обусловлена отсутствием малого параметра, разложение по которому гарантировало бы достаточную точность исходного приближения и малость поправок к этому приближению. Обилие работ, в которых используются те или иные приближенные уравнения для корреляционных функций жидкости, не решает проблемы, так как невозможно установить точность этих уравнений. Эта трудность в полной мере относится и к проблеме построения теории поверхностного натяжения жидкостей. В книгах [1] приведено замкнутое выражение для поверхностного натяжения простой жидкости через анизотропную часть бинарной корреляционной функции, отличной от нуля в переходном межфазном слое, однако для ее определения не существует регулярного метода.

Возможен другой подход - разложение поверхностной свободной энергии по корреляционным функциям объемных фаз, которые могут быть определены либо экспериментально, либо из некоторых приближенных уравнений. Разумеется, таким путем может быть получено только приближенное значение поверхностного натяжения, однако при этом остается возможность его уточнения путем учета следующих членов разложения.

\footnotetext{
*Тюменский государственный университет, Тюмень, Россия. E-mail: earin@utmn.ru
} 
Полная задача исследования свойств двухфазной системы требует определения термодинамических свойств и структурных (корреляционных) функций обеих объемных фаз, их расположения в пространстве и термодинамического потенциала и структуры переходного (поверхностного) слоя. При построении теории поверхностного натяжения примем естественное предположение, что первая часть задачи решена, т.е. все свойства объемных фаз известны. Расположение этих фаз в пространстве (и геометрическое положение поверхностного слоя) мы будем задавать, исходя из идеологии квазисредних Боголюбова [2], а именно полагая, что геометрия системы определяется внешним полем, настолько малым, что оно не влияет на термодинамические свойства как объемных фаз, так и поверхности.

То обстоятельство, что термодинамические параметры и корреляционные функции объемных фаз не являются независимыми, а связаны условиями фазового равновесия, позволяет, как будет показано ниже, варьировать в некоторых пределах разложение поверхностной энергии по структурным функциям объемных фаз.

\section{2. ВЫДЕЛЕНИЕ ПОВЕРХНОСТНОЙ ЭНЕРГИИ}

Все термодинамические и структурные функции системы определяются из вариационной задачи для термодинамического потенциала как функционала от всех частичных плотностей или корреляций [3]. Производящий функционал корреляционных функций $W(u)=\ln L(u)$, где $L(u)=Z(u) / Z(f)$ - введеный Боголюбовым [4] производящий функционал частичных плотностей, дает изменение термодинамического потенциала $\delta \Omega$ большого ансамбля, умноженного на обратную температуру $\beta$, в зависимости от дополнительного внешнего поля $\ln (1+u)=-\beta \phi$. Функциональное преобразование Лежандра

$$
W_{\rho}(\rho)=W(u)+\int\left(\rho(q) \ln \frac{\rho(q)}{e z(1+u(q))}-\rho_{0}(q) \ln \frac{\rho_{0}(q)}{e z}\right) d q
$$

определяет производящий функционал функций $W_{n}(\{n\})=\delta^{n} W_{\rho} /\left(\Pi_{n} \delta \rho(i)\right)$. Так как, в частности, функция $W_{2}(1,2)=C(1,2)$ совпадает с прямой корреляционной функцией Орнштейна-Цернике, то функции $W_{n}(\{n\})$ можно назвать обобщенными, или высшими прямыми корреляционными функциями.

Изменение термодинамического потенциала в зависимости от изменения унарной плотности приобретает вид [3]

$$
\beta\left(\Omega(\rho)-\Omega\left(\rho_{0}\right)\right)=\int\left(\rho(q) \ln \frac{\rho(q)}{e z(1+u(q))}-\rho_{0}(q) \ln \frac{\rho_{0}(q)}{e z}\right) d q-W_{\rho}(\rho) .
$$

Вариационный принцип для функционала $\Omega(\rho(q))$ определяет обратное преобразование Лежандра и дает уравнение для унарной плотности:

$$
-\beta \frac{d p}{d \rho}=\ln \frac{\rho(1)}{z^{*}}-W_{1}(1 \mid \rho(q))=0, \quad z^{*}=z(1+u(1)) .
$$

Равновесие двух фаз означает существование двух решений $\rho_{1}$ и $\rho_{2}$ этого уравнения при фиксированном значении $z^{*}$. 
Термодинамический потенциал системы жидкость-пар складывается из потенциалов объемных фаз и поверхностной свободной энергии:

$$
\Omega(\rho)=\Omega_{1}\left(\rho_{1}\right)+\Omega_{2}\left(\rho_{2}\right)+\Omega_{s},
$$

где $\rho_{1}$ и $\rho_{2}$ - унарные плотности объемных фаз, являющиеся в рассматриваемом случае постоянными,

$$
\Omega_{i}\left(\rho_{i}\right)=\int_{V_{i}} \omega\left(\rho_{i}\right) d(1)=-p_{i} V_{i},
$$

плотность термодинамического потенциала равна давлению в соответствующей фазе с обратным знаком: $\omega\left(\rho_{i}\right)=-p_{i}$.

Условия равновесия фаз при заданной температуре следуют из условия минимума потенциала (3) и сводятся к стандартным требованиям равенства химических потенциалов фаз и условию $p_{2}-p_{1}=\sigma\left(R_{1}^{-1}+R_{2}^{-1}\right)$ для разности давлений [5].

Унарная плотность двухфазной системы может быть представлена в виде

$$
\rho(q)=\rho_{1}+\Delta \rho f(q)=\rho_{2}-\Delta \rho(1-f(q))=\rho_{1}(1-f(q))+\rho_{2} f(q),
$$

где $\Delta \rho=\rho_{2}-\rho_{1}$ и структурная функция переходного (поверхностного) слоя $f(q)$ должна быть определена из вариационного принципа для термодинамического потенциала (3) при фиксированных значениях плотностей фаз $\rho_{1}$ и $\rho_{2}$, а следовательно, и активности $z$. При этом согласно уравнению (2)

$$
-\beta \frac{d p}{d \rho}=0, \quad \frac{d \ln z^{*}}{d \rho}=\frac{1}{\rho}-\frac{d W_{1}}{d \rho}, \quad-\beta \frac{d^{2} p}{d \rho^{2}}=\frac{1}{\rho}-\frac{d W_{1}}{d \rho}=\beta \frac{d p}{d \rho}+\frac{d \ln z^{*}}{d \rho}=\frac{\beta k}{\rho},
$$

где $k=k_{T}$ - обратная изотермическая сжимаемость $(\rho d p / d \rho)_{T}$, не зависящая от активности $z^{*}$. Старшие производные $d^{n} p / d \rho^{n}, n>2$, являются производными от величины $k(\rho)$.

Представление (4) симметрично относительно перестановки $\rho_{1} \Longleftrightarrow \rho_{2}$ и $f(q) \Longleftrightarrow$ $(1-f(q))$. Асимметрия переходного слоя возникает вследствие различия корреляционных функций фаз.

Очевидно, $f=0$ в объеме первой фазы и $1-f=0$ в объеме второй фазы; следовательно, термодинамический потенциал каждой фазы, заключенной в своем объеме, может быть представлен в виде

$$
\begin{aligned}
& \Omega_{1}\left(\rho_{1}\right)=\int_{V_{1}} \omega\left(\rho_{1}\right) d(1)=\int(1-f(1)) \omega\left(\rho_{1}\right) d(1), \\
& \Omega_{2}\left(\rho_{2}\right)=\int_{V_{2}} \omega\left(\rho_{2}\right) d(1)=\int f(1) \omega\left(\rho_{2}\right) d(1) .
\end{aligned}
$$

Тогда для поверхностной свободной энергии (поверхностного термодинамического потенциала) получим выражение

$$
\begin{aligned}
\Omega_{s} & =\int\left(\omega(\rho(1))-\omega\left(\rho_{1}\right)(1-f(1))-\omega\left(\rho_{2}\right) f(1)\right) d(1)= \\
& =\int\left(\left(\omega(\rho(1))-\omega\left(\rho_{1}\right)\right)(1-f(1))+\left(\omega(\rho(1))-\omega\left(\rho_{2}\right)\right) f(1)\right) d(1) .
\end{aligned}
$$


Очевидно, что подынтегральное выражение в формуле (5) отлично от нуля только в переходном межфазном слое; следовательно, это выражение пропорционально площади межфазной поверхности и действительно является поверхностной энергией.

Предположение, что давление $p(\rho)=-\omega(\rho)$ является локальной функцией плотности, не дает возможности построить какое-либо приближение для теории поверхностного натяжения жидкости. Действительно, в этом случае вариационная задача сводится к уравнению

$$
\frac{\delta \Omega}{\delta f(q)}=p\left(\rho_{2}\right)-p\left(\rho_{1}\right)-\left(\rho_{2}-\rho_{1}\right) \frac{d p(\rho)}{d \rho}=0,
$$

из которого невозможно определить зависимость от координат плотности $\rho(q)$ или структурной функции $f(q)$.

Для определения поверхностного натяжения необходимо учесть нелокальный характер зависимости давления от плотности. Очевидно, нелокальная часть термодинамического потенциала содержится в функционале $W_{\rho}$ и явно может быть представлена в виде разложения этого функционала по корреляционным функциям объемных фаз. Такое разложение можно получить из представления (5).

Для сокращения выкладок введем операторы $\delta_{1}$ и $\delta_{2}$, действующие на функционалы от унарной плотности по правилам

$$
\begin{aligned}
& \delta_{1} \Psi[\rho(q)]=\Delta \rho \int f(q) \frac{\delta \Psi}{\delta \rho(q)} d q \\
& \delta_{2} \Psi[\rho(q)]=-\Delta \rho \int(1-f(q)) \frac{\delta \Psi}{\delta \rho(q)} d q .
\end{aligned}
$$

Тогда $\Omega(\rho(q))=e^{\delta_{1}} \Omega\left(\rho_{1}\right)=e^{\delta_{2}} \Omega\left(\rho_{2}\right)$ и $\Omega\left(\rho_{2}\right)=e^{\delta_{1}-\delta_{2}} \Omega\left(\rho_{1}\right)$, а также $\Omega\left(\rho_{1}\right)=e^{-\delta_{1}} \times$ $\Omega(\rho(\rho)), \quad \Omega\left(\rho_{2}\right)=e^{-\delta_{2}} \Omega(\rho(\rho))$.

Разложение функционала $\Omega(\rho)$ имеет вид интегростепенного ряда с симметричными ядрами. Ограничение области интегрирования по последней переменной для членов такого ряда осуществляется формально оператором $\left(\delta_{2}-\delta_{1}\right)^{-1} \delta_{2}$ для области $V_{1}$ и оператором $\left(\delta_{1}-\delta_{2}\right)^{-1} \delta_{1}$ для области $V_{2}$. Тогда

$$
\begin{aligned}
\Omega_{s} & =\Omega(\rho(q))-\frac{\delta_{2} \Omega\left(\rho_{1}\right)}{\delta_{2}-\delta_{1}}-\frac{\delta_{1} \Omega\left(\rho_{2}\right)}{\delta_{1}-\delta_{2}}=\left(1-\frac{\delta_{2} e^{-\delta_{1}}}{\delta_{2}-\delta_{1}}-\frac{\delta_{1} e^{-\delta_{2}}}{\delta_{1}-\delta_{2}}\right) \Omega(\rho(q))= \\
& =\frac{\left(1-e^{-\delta_{2}}\right) \delta_{1}-\left(1-e^{-\delta_{1}}\right) \delta_{2}}{\delta_{1}-\delta_{2}} \Omega(\rho(q)) .
\end{aligned}
$$

Из представления (6) сразу следует разложение поверхностной энергии по корреляционным функциям переходного слоя. Для того чтобы перейти к разложению по корреляционным функциям объемных фаз, выразим функционал $\Omega(\rho(q))$ либо через $\Omega\left(\rho_{1}\right)$, либо через $\Omega\left(\rho_{2}\right)$, либо через линейную комбинацию этих функционалов $t \Omega\left(\rho_{1}\right)+(1-t) \Omega\left(\rho_{2}\right)$ при любом $0 \leqslant t \leqslant 1$. Так как

$$
t \Omega\left(\rho_{1}\right)+(1-t) \Omega\left(\rho_{2}\right)=\left(t e^{-\delta_{1}}+(1-t) e^{-\delta_{2}}\right) \Omega(\rho(q)),
$$

то

$$
\Omega(\rho(q))=e^{\left(\delta_{1}+\delta_{2}\right) / 2}\left(t e^{\left(\delta_{2}-\delta_{1}\right) / 2}+(1-t) e^{\left(\delta_{1}-\delta_{2}\right) / 2}\right)^{-1}\left(t \Omega\left(\rho_{1}\right)+(1-t) \Omega\left(\rho_{2}\right)\right) .
$$


Это соотношение определяет разложение и термодинамического потенциала, и всех корреляционных функций двухфазной системы по корреляционным функциям объемных фаз и структурной функции переходного слоя. Формально

$$
\begin{gathered}
\left(t e^{\left(\delta_{2}-\delta_{1}\right) / 2}+(1-t) e^{\left(\delta_{1}-\delta_{2}\right) / 2}\right)^{-1}\left(t \Omega\left(\rho_{1}\right)+(1-t) \Omega\left(\rho_{2}\right)\right)= \\
=e^{-\left(\delta_{1}+\delta_{2}\right) / 2} \Omega(\rho(q))=\Omega\left(\frac{\rho_{1}+\rho_{2}}{2}\right)=\Omega(\bar{\rho}) .
\end{gathered}
$$

При однородной плотности $\rho=\bar{\rho}=\left(\rho_{1}+\rho_{2}\right) / 2$ термодинамический потенциал неустойчив, однако его значение может быть получено как аналитическое продолжение из областей устойчивых состояний при $\rho=\rho_{1}$ и $\rho=\rho_{2}$, в частности, разложением по разности плотностей фаз $\left(\rho_{2}-\rho_{1}\right)$. Возможность этого аналитического продолжения следует из термодинамического равновесия фаз, из того факта, что оба устойчивых состояния (и их плотности) определяются как два различных минимума единого функционала, разделенные максимумом, в окрестности которого однородная система неустойчива. В дальнейшем выражение $F\left(\left(\rho_{1}+\rho_{2}\right) / 2\right)=F(\bar{\rho})$ будет использоваться для краткой записи разложения $t F\left(\rho_{1}\right)+(1-t) F\left(\rho_{2}\right)$ - выпуклой оболочки функций $F\left(\rho_{1}\right)$ и $F\left(\rho_{2}\right)$ по разности плотностей $\rho_{2}-\rho_{1}=\Delta \rho$.

Полагая $t=1$, получим разложение поверхностной свободной энергии по корреляционным функциям первой фазы, а полагая $t=0$ - по корреляционным функциям второй фазы. Кроме того, выбрав соответствующее значение параметра $t$, можно построить разложение симметричное, либо учитывающее различное влияние фаз на исследуемое свойство переходного слоя.

Необходимо подчеркнуть, что возможность подобной неоднозначности разложения поверхностной энергии определяется тем, что существование нескольких фаз, находящихся в равновесии, означает существование такого же количества минимумов единого функционала термодинамического потенциала, что позволяет формально связать свойства всех фаз и всей многофазной системы. Существование такого функционала следует из общих свойств статистической суммы, приведенных, например, в монографии [6].

\section{3. РАЗЛОЖЕНИЕ ПОВЕРХНОСТНОЙ ЭНЕРГИИ}

Будем предполагать, что свойства объемных фаз известны, и можно использовать связи, формально выраженные приведенными выше соотношениями (5)-(7). В итоге разложение поверхностной свободной энергии по структурным функциям объемных фаз примет вид

$$
\begin{aligned}
\beta \Omega_{s}=\int & \left(\rho(q) \ln \rho(q)-(1-f(q)) \rho_{1} \ln \rho_{1}-f(q) \rho_{2} \ln \rho_{2}\right) d q- \\
& -\left(e^{\left(\delta_{1}+\delta_{2}\right) / 2}-\operatorname{ch}\left(\frac{\delta_{1}-\delta_{2}}{2}\right)-\frac{\delta_{1}+\delta_{2}}{\delta_{1}-\delta_{2}} \operatorname{sh}\left(\frac{\delta_{1}-\delta_{2}}{2}\right)\right) W_{\rho}(\bar{\rho}) .
\end{aligned}
$$


Используем соотношения

$$
\begin{aligned}
e^{\left(\delta_{1}+\delta_{2}\right) / 2} & =\operatorname{ch}\left(\frac{\delta_{1}+\delta_{2}}{2}\right)+\left(\delta_{1}+\delta_{2}\right) \frac{\operatorname{sh}\left(\left(\delta_{1}+\delta_{2}\right) / 2\right)}{\delta_{1}+\delta_{2}}, \\
& \left(\frac{\delta_{1}+\delta_{2}}{2}\right)^{2}=\left(\frac{\delta_{1}-\delta_{2}}{2}\right)^{2}+\delta_{1} \delta_{2}
\end{aligned}
$$

Функции $\operatorname{ch}(x)$ и $\operatorname{sh}(x) / x$ являются целыми функциями от $x^{2}$. Для них справедливо разложение

$$
F\left(x^{2}+y\right)=\sum_{0}^{\infty} \frac{y^{n}}{2^{n} n !}\left(\frac{d}{x d x}\right)^{n} F\left(x^{2}\right) .
$$

Введем систему функций

$$
\psi_{n}(x)=\left(\frac{d}{x d x}\right)^{n} \psi_{0}(x), \quad \psi_{0}(x)=\operatorname{ch}(x) .
$$

Эти функции можно связать с функциями Бесселя полуцелого индекса от мнимого аргумента [7] и получить для них интегральное представление

$$
\psi_{n}(x)=\int_{0}^{1} t_{n} d t_{n} \int_{0}^{t_{n}} t_{n-1} d t_{n-1} \ldots \int_{0}^{t_{2}} \operatorname{ch}\left(t_{1} x\right) d t_{1}=\int_{0}^{1} \frac{\left(1-t^{2}\right)^{n-1}}{2^{n-1}(n-1) !} \operatorname{ch}(t x) d t .
$$

В частности,

$$
\psi_{1}(x)=\int_{0}^{1} \operatorname{ch}(t x) d t=\frac{\operatorname{sh} x}{x}, \quad \psi_{2}(x)=\frac{\operatorname{ch} x}{x^{2}}-\frac{\operatorname{sh} x}{x^{3}} .
$$

Тогда

$$
\begin{aligned}
e^{\left(\delta_{1}+\delta_{2}\right) / 2} & -\operatorname{ch}\left(\frac{\delta_{1}-\delta_{2}}{2}\right)-\frac{\delta_{1}+\delta_{2}}{\delta_{1}-\delta_{2}} \operatorname{sh}\left(\frac{\delta_{1}-\delta_{2}}{2}\right)= \\
& =\sum_{1}^{\infty} \frac{\left(\delta_{1} \delta_{2}\right)^{n}}{2^{n} n !}\left(\psi_{n}\left(\frac{\delta_{1}-\delta_{2}}{2}\right)+\frac{\delta_{1}+\delta_{1}}{2} \psi_{n+1}\left(\frac{\delta_{1}-\delta_{2}}{2}\right)\right) .
\end{aligned}
$$

В итоге разложение поверхностной энергии приобретает вид

$$
\begin{aligned}
\beta \Omega_{s}=\int & \left(\rho(q) \ln \rho(q)-(1-f(q)) \rho_{1} \ln \rho_{1}-f(q) \rho_{2} \ln \rho_{2}\right) d q- \\
& -\sum_{1}^{\infty} \frac{\left(\delta_{1} \delta_{2}\right)^{n}}{2^{n} n !}\left(\psi_{n}\left(\frac{\delta_{1}-\delta_{2}}{2}\right)+\frac{\delta_{1}+\delta_{1}}{2} \psi_{n+1}\left(\frac{\delta_{1}-\delta_{2}}{2}\right)\right) W_{\rho}(\bar{\rho}) .
\end{aligned}
$$

При этом

$$
\begin{aligned}
& 2 \operatorname{ch}\left(\frac{\delta_{1}-\delta_{2}}{2}\right) W_{s}(\{s\} \mid \bar{\rho})=W_{s}\left(\{s\} \mid \rho_{1}\right)+W_{s}\left(\{s\} \mid \rho_{2}\right), \\
& 2 \operatorname{sh}\left(\frac{\delta_{1}-\delta_{2}}{2}\right) W_{s}(\{s\} \mid \bar{\rho})=W_{s}\left(\{s\} \mid \rho_{2}\right)-W_{s}\left(\{s\} \mid \rho_{1}\right) .
\end{aligned}
$$


Разложение (11) содержит положительные степени оператора $\left(\delta_{1}-\delta_{2}\right)$, осуществляющего разложение функционала $W_{\rho}$ и функций $W_{s}$ по прямым корреляционным функциям, оператор $\left(\delta_{1}+\delta_{2}\right)$, вносящий антисимметричный при перестановке фаз множитель $(2 f(q)-1)$, и, что наиболее существенно, оператор $\left(\delta_{1} \delta_{2}\right)$, вносящий множитель $f\left(q_{1}\right)\left(1-f\left(q_{2}\right)\right)$, ограничивающий область интегрирования как со стороны одной, так и со стороны другой фазы. Это важное свойство обеспечивается тем, что разложение (10) сохраняет симметрию операторов $\delta_{1}$ и $\delta_{2}$ в каждом члене.

Полученное разложение на первый взгляд вполне однозначно, однако если воспользоваться соотношением $W_{n}\left(\{n\} \mid \rho_{2}\right)=e^{\delta_{1}-\delta_{2}} W_{n}\left(\{n\} \mid \rho_{1}\right)$, то можно реализовать возможность разложения поверхностной энергии по произвольной линейной комбинации корреляционных функций объемных фаз.

Выражение $f(1)(1-f(2))$, проинтегрированное с симметричной по всем аргументам функцией $W_{n}(\{n\})$, может быть разделено на локальный вклад и нелокальную часть, для чего его следует преобразовать по правилу

$$
f(1)(1-f(2)) \longrightarrow f(1)(1-f(1))+\frac{(f(1)-f(2))^{2}}{2},
$$

которое следует из тождества

$$
2 f(1)(1-f(2))+f(2)-f(1)=f(1)(1-f(1))+f(2)(1-f(2))+(f(1)-f(2))^{2}
$$

и эквивалентности координат (1) и (2) при интегрировании.

Аналогично выражение $f(1)(1-f(2))(2 f(3)-1)$ приводится к виду

$$
\begin{aligned}
f(1)(1- & f(2))(2 f(3)-1) \longrightarrow f(1)(1-f(1))(2 f(1)-1)+ \\
& +3(f(1)-f(2))^{2} \frac{f(1)+f(2)-1}{2}+(f(2)-f(1))^{2}(f(3)-f(1)) .
\end{aligned}
$$

При этом локальные произведения $f(1)(1-f(1))$ и $f(1)(1-f(1))(2 f(1)-1)$ имеют первый порядок малости как по $f$ в области $f \rightarrow 0$, так и по $1-f$ в области $f \rightarrow 1$. В то же время выражение $(f(2)-f(1))^{2}$ вследствие малости эффективного радиуса корреляционных функций имеет второй порядок малости как в одной, так и во второй области.

Локальная часть старших (по степеням оператора $\left.\left(\delta_{1} \delta_{2}\right)\right)$ членов разложения (9) выделяется аналогично, хотя и более громоздко. Эта процедура осуществляется следующим образом.

В результате однократного применения оператора $\left(\delta_{1}-\delta_{2}\right)$ получим выражение $\left(\delta_{1}-\delta_{2}\right) W_{\rho}=\Delta \rho \int W_{1}(1) d(1)$. Следовательно, произведя замену

$$
\delta_{1} \longrightarrow f(1)\left(\delta_{1}-\delta_{2}\right), \quad \delta_{2} \longrightarrow(f(1)-1)\left(\delta_{1}-\delta_{2}\right),
$$


получим полный локальный вклад, следующий из разложения (10), в виде

$$
\begin{aligned}
\int d(1) \sum_{1}^{\infty} & \frac{\left(-(\Delta \rho)^{2} f(1)(1-f(1))\right)^{n}}{2^{n} n !} \times \\
& \times\left(\psi_{n}\left(\frac{\delta_{1}-\delta_{2}}{2}\right) \int W_{2 n}(1,\{2 n-1\} \mid \bar{\rho}) d\{2 n-1\}+\right. \\
& \left.+\frac{\Delta \rho(2 f(1)-1)}{2} \psi_{n+1}\left(\frac{\delta_{1}-\delta_{2}}{2}\right) \int W_{2 n+1}(1,\{2 n\} \mid \bar{\rho}) d\{2 n\}\right) .
\end{aligned}
$$

Объединяя полученное разложение с первым членом формул (9), (11), получим локальную часть поверхностной энергии:

$$
\begin{aligned}
\int((1- & \left.f(q)) p\left(\rho_{1}\right)+f(q) p\left(\rho_{2}\right)-p_{\text {loc }}(\rho(q))\right) d q= \\
= & -\int d(q) \sum_{1}^{\infty} \frac{(-2 f(q)(1-f(q)))^{n}}{n !} \times \\
& \times\left(\Theta^{2 n} \psi_{n}(\Theta)+(2 f(q)-1) \Theta^{2 n+1} \psi_{n+1}(\Theta)\right) p(\bar{\rho}), \quad \Theta=\frac{\Delta \rho}{2} \frac{d}{d \bar{\rho}},
\end{aligned}
$$

где $p_{\text {loc }}(\rho)$ - аналитическое продолжение функций однородных средних плотностей $p\left(\rho_{1}\right)$ и $p\left(\rho_{2}\right)$ при $z^{*}=$ const на значение плотности, лежащей в интервале $\left(\rho_{1}, \rho_{2}\right)$ и содержащей функцию $f(q)$, согласно уравнению (4), как параметр. Локальные члены первого и второго порядков с учетом значений производных $d p / d \rho$ и $d^{2} p / d \rho^{2}$ принимают вид

$$
\begin{aligned}
& \frac{(\Delta \rho)^{2}}{4} \int d q(f(q)(1-f(q)))^{2}\left(\frac{k_{2}}{\rho_{2}} f(q)+\frac{k_{1}}{\rho_{1}}(1-f(q))\right)+\cdots- \\
& \quad-\left(p\left(\rho_{2}\right)-p\left(\rho_{1}\right)\right) \int d q(2 f(q)-1)\left(f(q)(1-f(q))-3 f^{2}(q)(1-f(q))^{2}+\cdots\right) .
\end{aligned}
$$

В важном случае плоской границы, который будет рассмотрен прежде всего, $\left(p\left(\rho_{2}\right)-\right.$ $\left.p\left(\rho_{1}\right)\right)=0$ и второй член отсутствует.

Для выделения нелокальной части поверхностной энергии используем интегральное представление функций $\psi_{n}(x)$. Явное выражение членов порядка $n$, как локальных, так и нелокальных, требует учета вклада от операторов $\left(\delta_{1} \delta_{2}\right)^{k}$ для всех $k \leqslant n$.

Нелокальные члены второго порядка, входящие в выражения (10), (11), при $\left(\delta_{1} \delta_{2}\right)^{1}$ и $\left(\delta_{1} \delta_{2}\right)^{2}$ дают вклад

$$
\frac{(\Delta \rho)^{2}}{4} \int(f(2)-f(1))^{2}\left((1-f(1)) W_{2}\left(1,2 \mid \rho_{1}\right)+f(1) W_{2}\left(1,2 \mid \rho_{2}\right)\right) d(1,2) .
$$

Очевидно, что $f(1)=f(x), f(2)=f(x+h)$, где $x$ - координата, нормальная к разделяющей поверхности, и $h$ - нормальная проекция расстояния между точками (1) и (2). Вводя цилиндрические координаты $h, r_{1}, \varphi, r^{2}=h^{2}+r_{1}^{2}$, получим

$$
\int_{0}^{2 \pi} d \varphi \int_{0}^{\infty} r_{1} d r_{1} W_{2}\left(r \mid \rho_{i}\right)=2 \pi \int_{h}^{\infty} W_{2}\left(r \mid \rho_{i}\right) r d r=K_{i}(h),
$$


причем $K_{i}(-h)=K_{i}(h)$. Кроме того, $\int d(1)=S \int d x$, где $S$ - площадь поверхностного слоя. Таким образом, для поверхностного натяжения получим (в случае плоской границы) разложение

$$
\begin{aligned}
\sigma=\frac{\Omega_{s}}{S} & =\frac{(\Delta \rho)^{2}}{4} \int(f(x)(1-f(x)))^{2}\left(\frac{k_{2}}{\rho_{2}} f(x)+\frac{k_{1}}{\rho_{1}}(1-f(x))\right) d x+ \\
& +\frac{(\Delta \rho)^{2}}{4 \beta} \int(f(x+h)-f(x))^{2}\left((1-f(x)) K_{1}(h)+f(x) K_{2}(h)\right) d h d x+\cdots .
\end{aligned}
$$

Члены более высокого порядка весьма громоздки, хотя процедура их получения очевидна.

Выражение для поверхностного натяжения простой жидкости как функционала от структурной функции переходного слоя, отличающееся от функционала (16) членами более высокого порядка (не приведенными здесь ввиду их громоздкости), получено в работе [8] путем непосредственного разложения термодинамического потенциала поверхностного слоя по степеням структурной функции.

\section{4. ПЛОСКАЯ ГРАНИЦА РАЗДЕЛА}

В случае плоской межфазной границы разность давлений равна нулю и площадь границы раздела не зависит от координаты, нормальной к этой границе, что несколько упрощает анализ полученного выражения для поверхностного натяжения.

Функционал (16) содержит усредненную первую разность функции $f(x)$. Вариационный принцип для этого функционала $\delta \sigma / \delta f(x)=0$ приводит к нелинейному интегральному уравнению

$$
\begin{gathered}
f(x)(1-f(x))(1-2 f(x))\left((1-f(x)) \frac{\beta k_{1}}{\rho_{1}}+f(x) \frac{\beta k_{2}}{\rho_{2}}\right)+ \\
+\frac{1}{2} f^{2}(x)(1-f(x))^{2}\left(\frac{\beta k_{2}}{\rho_{2}}-\frac{\beta k_{1}}{\rho_{1}}\right)= \\
=\int(f(x+h)+f(x-h)-2 f(x))\left((1-f(x)) K_{1}(h)+f(x) K_{2}(h)\right) d h+ \\
+\frac{1}{2} \int(f(x)-f(x+h))^{2}\left(K_{2}(h)-K_{1}(h)\right) d x,
\end{gathered}
$$

правая часть которого содержит усредненную вторую разность функции $f(x)$. Так как $K(-h)=K(h)$, то в правой части опущено слагаемое

$$
\begin{gathered}
\int(f(x)-f(x+h))^{2}\left(K_{2}(h)-K_{1}(h)\right) d x-\int(f(x)-f(x-h))^{2}\left(K_{2}(h)-K_{1}(h)\right) d x= \\
=\int(f(x+h)+f(x-h)-2 f(x))(f(x+h)-f(x-h))\left(K_{2}(h)-K_{1}(h)\right) d x=0 .
\end{gathered}
$$

Уравнение (17) является аналогом уравнения Эйлера второго прядка стандартной задачи вариационного исчисления. Для того чтобы найти аналог первого интеграла 
Эйлера, умножим это уравнение на $f^{\prime}(x)$ и проинтегрируем от $-\infty$ до $x$. С учетом того, что $f(-\infty)=0$, получим

$$
\begin{aligned}
& \frac{1}{2}(f(x)(1-f(x)))^{2}\left(\frac{k_{1}}{\rho_{1}}(1-f(x))+\frac{k_{2}}{\rho_{2}} f(x)\right)= \\
& =2 \int_{0}^{\infty}\left((1-f(x)) K_{1}(h)+f(x) K_{2}(h)\right) d h \int_{0}^{h}(f(x)-f(x+t-h)) f^{\prime}(x+t) d t+ \\
& \quad+\frac{1}{2} \int_{0}^{\infty}\left(K_{2}(h)-K_{1}(h)\right) d h \int_{0}^{h}\left((f(x+t)-f(x))^{2} f^{\prime}(x+t-h)-\right. \\
& \left.\quad-(f(x)-f(x+t-h))^{2} f^{\prime}(x+t)\right) d t
\end{aligned}
$$

Уравнение (18) содержит первую усредненную разность функции $f(x)$.

Различные варианты итерационной процедуры расчета структурной функции переходного слоя $f(x)$ могут базироваться как на уравнении (17), так и на уравнении (18).

В частности, из уравнения (18) легко получить асимптотику функции $f(x)$ в области $x<0$, где $f(x) \ll 1$ и $|x|$ больше ширины переходного слоя. Полагая в нулевом приближении $f(x) \approx \vartheta(x)$, в первом приближении получим в этой области

$$
f(x) \approx \frac{4 \rho_{1}}{\beta k_{1}} \int_{|x|}^{\infty} K_{1}(h) d h .
$$

Воспользовавшись тождеством

$$
\int_{0}^{h}(f(x)-f(x+t-h)) f^{\prime}(x+t) d t=\int_{0}^{h}(f(x+t)-f(x)) f^{\prime}(x+t-h) d t,
$$

получим в области $x>0$ и $1-f(x) \ll 1$ асимптотику

$$
1-f(x) \approx \frac{4 \rho_{2}}{\beta k_{2}} \int_{x}^{\infty} K_{2}(h) d h
$$

по существу совпадающую с асимптотикой, приведенной в работе [9] (там же можно найти подробную библиографию работ Куни и Русанова, посвященных исследованию асимптотики структуры поверхностного слоя простой жидкости).

\section{5. ПРОСТЫЕ ОЦЕНКИ}

Простейшие оценки поверхностного натяжения легко провести для области, в которой выполняются условия справедливости теории Ландау. В этой области, достаточно близкой к критической точке, ширина переходного слоя может быть больше эффективного радиуса прямой корреляции, остающегося конечным и в критической точке, поэтому можно использовать аппроксимацию $f(x+h)-f(x) \approx f^{\prime}(x) h$. Полагая

$$
\int h^{2} K(h) d h=\frac{1}{3} \int r^{2} W_{2}(r) d^{3} r=\tilde{h}^{2}
$$


получим

$$
\begin{aligned}
\frac{4 \beta}{(\Delta \rho)^{2}} \sigma \approx \int & \left(\frac{\beta k_{1}}{\rho_{1}}(1-f(x))+\frac{\beta k_{2}}{\rho_{2}} f(x)\right)(f(x)(1-f(x)))^{2} d x+ \\
& +\int\left(\tilde{h}_{1}^{2}(1-f(x))+\tilde{h}_{2}^{2} f(x)\right) f^{\prime 2}(x) d x .
\end{aligned}
$$

Вариационная задача для этого функционала имеет первый интеграл Эйлера (при $\left.f^{\prime}( \pm \infty)=0\right)$

$$
\left(\frac{\beta k_{1}}{\rho_{1}}(1-f(x))+\frac{\beta k_{2}}{\rho_{2}} f(x)\right)(f(x)(1-f(x)))^{2}=\left(\tilde{h}_{1}^{2}(1-f(x))+\tilde{h}_{2}^{2} f(x)\right) f^{\prime 2}(x),
$$

откуда

$$
\frac{d}{d x} \ln \frac{1-f(x)}{f(x)}= \pm\left(\frac{\beta k_{1}}{\rho_{1}}(1-f(x))+\frac{\beta k_{2}}{\rho_{2}} f(x)\right)^{1 / 2}\left(\tilde{h}_{1}^{2}(1-f(x))+\tilde{h}_{2}^{2} f(x)\right)^{-1 / 2} .
$$

Функцию $f(x)$ можно найти отсюда последовательными итерациями. Если можно пренебречь малой разностью параметров фаз и принять для них некоторые средние значения, то

$$
f(x)=\left(e^{ \pm x / \lambda}+1\right)^{-1}, \quad \lambda^{2}=\frac{\tilde{h}^{2} \rho}{\beta k} .
$$

Знак выбирается в зависимости от выбора направления изменения координаты. В результате для величины поверхностного натяжения получим оценку

$$
\sigma \approx \frac{(\Delta \rho)^{2} \tilde{h}}{6}\left(\frac{k}{\beta \rho}\right)^{1 / 2}
$$

что находится в удовлетворительном согласии с экспериментальными данными и уточненным правилом парахора $\sigma \sim(\Delta \rho)^{11 / 3}$, если принять оценки $\Delta \rho \sim\left(T_{\mathrm{c}}-T\right)^{0.36}$, $k \sim\left(T_{\mathrm{c}}-T\right)^{1.18}$ с наиболее вероятными значениями показателей [10]. При реализации итерационной процедуры уточнения решения выражение (19) может быть принято за исходную функцию.

Область применимости решения (19) можно расширить, определяя $\lambda$ как вариационный параметр функционала (16) прямым методом.

Переходный (поверхностный) слой можно определить как область, где $f^{\prime} \neq 0$. Ширина решения (19) равна $\lambda$, так как $f^{\prime}=4 \lambda^{-1} \mathrm{ch}^{-2}(x /(2 \lambda))$. Фурье-образ этой функции равен $2 \pi \lambda \xi \mathrm{sh}^{-1}(\pi \lambda \xi)$, т.е. ширина фурье-образа пространственной неоднородности равна $1 / \lambda$, причем фурье-компоненты сосредоточены в окрестности нуля пространства волновых чисел $\xi$. В критической точке изотермическая сжимаемость бесконечна, $k_{T} \rightarrow 0$, следовательно, $\lambda \rightarrow \infty, 1 / \lambda \rightarrow 0$. Это очевидное свойство связано с нарушением условия устойчивости однородного состояния. Вариация термодинамического потенциала однородной системы при неоднородном возмущении плотности равна $\int\left(1-(2 \pi)^{3 / 2} \rho \widetilde{W}_{2}(\xi)\right)|\widetilde{\delta} \rho(\xi)|^{2} d^{3} \xi$, где $\widetilde{\delta} \rho(\xi)$ - фурье-компонента возмущения плотности и $1-(2 \pi)^{3 / 2} \rho W_{2}(\xi)$ - структурный фактор жидкости (однородной системы в общем случае), положительность которого обеспечивает устойчивость однородной системы. $\mathrm{B}$ критической точке $\beta k_{T}=1-\rho \int W_{2}(r) d^{3} r=$ 
$\left.1-(2 \pi)^{3 / 2} \rho \widetilde{W}_{2}(0)\right) \rightarrow 0$, ниже критической точки условие устойчивости нарушается в некоторой области волновых чисел в окрестности нуля, что и приводит к тому, что устойчивость системы обеспечивается возникновением пространственной неоднородности $(\widetilde{\delta}(\xi) \neq 0)$ шириной $\lambda$ в координатном пространстве и $\lambda^{-1}$ в пространстве волновых чисел, имеющей порядок ширины области, в которой $1-(2 \pi)^{3 / 2} \rho \widetilde{W}_{2}(\xi)<0$.

Следует еще раз подчеркнуть, что уточнение выражения (16) для поверхностного натяжения может быть проведено тем же методом, который был изложен при получении вклада второго порядка, но потребует весьма громоздких выкладок. Фрагменты вкладов третьего и четвертого порядков входят в член с $n=2$ рядов (10), (11), но полное и правильное выражение вклада указанных порядков требует учета членов рядов (10), (11) с $n=3$ и $n=4$, соответственно.

Ясно, что аналитическое решение вариационной задачи с учетом членов высокого порядка будет труднообозримым, поэтому более точные приближения следует находить численными методами.

\section{ПРИЛОЖЕНИЕ}

Метод выделения нелокальной части из члена разложений (10), (11) с $n=1$ был приведен в тексте. Выделение нелокального вклада второго порядка из члена с $n=2$ проводится в несколько этапов. На первом этапе

$$
\begin{aligned}
& f(1)(1-f(2)) \longrightarrow f(1)(1-f(1))+\frac{(f(1)-f(2))^{2}}{2}, \\
& f(3)(1-f(4)) \longrightarrow f(3)(1-f(3))+\frac{(f(3)-f(4))^{2}}{2} .
\end{aligned}
$$

Опуская члены порядков выше второго, получим

$$
f(1)(1-f(1)) f(3)(1-f(3)) \longrightarrow f^{2}(1)(1-f(1))^{2}-\frac{(f(1)(1-f(1))-f(2)(1-f(2)))^{2}}{2} ;
$$

наконец,

$$
\begin{aligned}
& -\frac{(f(1)(1-f(1))-f(2)(1-f(2)))^{2}}{2}= \\
& \quad=-\frac{(f(1)-f(2))^{2}\left(1-2 f(1)(1-f(1))-2 f(2)(1-f(2))-(f(1)-f(2))^{2}\right)}{2}= \\
& =-\frac{(f(1)-f(2))^{2}}{2}+\cdots .
\end{aligned}
$$

Член разложения (11) с $n=1$ можно записать как

$$
-\frac{\delta_{1} \delta_{2}}{2} \psi_{1}\left(\frac{\delta_{1}-\delta_{2}}{2}\right) W_{\rho}(\bar{\rho})-\frac{\delta_{1} \delta_{2}\left(\delta_{1}+\delta_{2}\right)}{4} \psi_{2}\left(\frac{\delta_{1}-\delta_{2}}{2}\right) W_{\rho}(\bar{\rho}) .
$$

Нелокальный вклад первого слагаемого имеет вид

$$
\frac{(\Delta \rho)^{2}}{8} \int_{0}^{1} d t \int(f(1)-f(2))^{2}\left(W_{2}\left(1,2 \mid \bar{\rho}+\frac{t \Delta \rho}{2}\right)+W_{2}\left(1,2 \mid \bar{\rho}-\frac{t \Delta \rho}{2}\right)\right) d(1,2) ;
$$


второе слагаемое дает нелокальный член второго порядка

$$
\begin{aligned}
& \frac{3(\Delta \rho)^{2}}{8} \int_{0}^{1} d t \int(f(1)-f(2))^{2}(2 f(1)-1) \times \\
& \quad \times\left(W_{2}\left(1,2 \mid \bar{\rho}+\frac{t \Delta \rho}{2}\right)-W_{2}\left(1,2 \mid \bar{\rho}-\frac{t \Delta \rho}{2}\right)\right) d(1,2)
\end{aligned}
$$

и член третьего порядка.

Член с $n=2$ разложения (11) имеет вид

$$
-\frac{\left(\delta_{1} \delta_{2}\right)^{2}}{8} \psi_{2}\left(\frac{\delta_{1}-\delta_{2}}{2}\right) W_{\rho}(\bar{\rho})-\frac{\left(\delta_{1} \delta_{2}\right)^{2}\left(\delta_{1}+\delta_{2}\right)}{16} \psi_{3}\left(\frac{\delta_{1}-\delta_{2}}{2}\right) W_{\rho}(\bar{\rho}) .
$$

Первое слагаемое дает нелокальный вклад второго порядка

$$
\begin{aligned}
\frac{(\Delta \rho)^{4}}{64} \int_{0}^{1} \int\left(W_{4}\left(1,2,3,4 \mid \bar{\rho}+\frac{t \Delta \rho}{2}\right)+W_{4}\left(1,2,3,4 \mid \bar{\rho}-\frac{t \Delta \rho}{2}\right)\right) \times \\
\quad \times(f(1)-f(2))^{2} d(1,2,3,4)\left(1-t^{2}\right) d t= \\
=\frac{(\Delta \rho)^{2}}{16} \int_{0}^{1}\left(1-t^{2}\right) d t \frac{d^{2}}{d t^{2}} \int\left(W_{2}\left(1,2 \mid \bar{\rho}+\frac{t \Delta \rho}{2}\right)+\right. \\
\left.\quad+W_{2}\left(1,2 \mid \bar{\rho}-\frac{t \Delta \rho}{2}\right)\right)(f(1)-f(2))^{2} d(1,2)= \\
=\frac{(\Delta \rho)^{2}}{8} \int\left(W_{2}\left(1,2 \mid \rho_{1}\right)+W_{2}\left(1,2 \mid \rho_{2}\right)\right)(f(1)-f(2))^{2} d(1,2)- \\
\quad-\frac{(\Delta \rho)^{2}}{8} \int_{0}^{1} d t \int(f(1)-f(2))^{2} \times \\
\quad \times\left(W_{2}\left(1,2 \mid \bar{\rho}+\frac{t \Delta \rho}{2}\right)+W_{2}\left(1,2 \mid \bar{\rho}-\frac{t \Delta \rho}{2}\right)\right) d(1,2) .
\end{aligned}
$$

Аналогично во второе слагаемое входит комбинация

$$
\int_{0}^{1}\left(1-t^{2}\right)^{2} \frac{d^{3}}{d t^{3}}\left(W_{2}\left(1,2 \mid \bar{\rho}+\frac{t \Delta \rho}{2}\right)-W_{2}\left(1,2 \mid \bar{\rho}-\frac{t \Delta \rho}{2}\right)\right) d t
$$

что приводит к нелокальному вкладу второго порядка

$$
\begin{aligned}
\frac{(\Delta \rho)^{2}}{8} \int & \left(W_{2}\left(1,2 \mid \rho_{1}\right)-W_{2}\left(1,2 \mid \rho_{2}\right)\right)(f(1)-f(2))^{2}(2 f(1)-1) d(1,2)- \\
& -\frac{3(\Delta \rho)^{2}}{8} \int_{0}^{1} d t \int(f(1)-f(2))^{2}(2 f(1)-1) \times \\
& \times\left(W_{2}\left(1,2 \mid \bar{\rho}+\frac{t \Delta \rho}{2}\right)-W_{2}\left(1,2 \mid \bar{\rho}-\frac{t \Delta \rho}{2}\right)\right) d(1,2) .
\end{aligned}
$$

В совокупности все члены второго порядка дают выражение, входящее в формулу (16). Все члены с $n>2$ - и локальные, и нелокальные - имеют более высокий порядок. 


\section{Список литературы}

[1] С. Оно, С. Кондо, Молекулярная теория поверхностного натяжения в жидкостях, ИЛ, М., 1963; К. Крокстон, Физика жидкого состояния. Статистическое введение, Мир, М., 1978.

[2] Н. Н. Боголюбов, Квазисредние в задачах статистической механики. Избранные трудъ, m. 3, Наукова думка, Киев, 1971.

[3] Э.А. Аринштейн, ТМФ, 124:1 (2000), 136-147; 141:1 (2004), 152-160.

[4] Н. Н. Боголюбов, Метод функционалъных производных в статистической механике. Избранные труды, m. 2, Наукова думка, Киев, 1970.

[5] Л. Д. Ландау, Е. М. Лифшиц, Теоретическая физика. T. V. Статистическая физика, Наука, М., 1976.

[6] Р. Балеску, Равновесная и неравновесная статистическая механика, m. 1, Мир, М., 1978.

[7] Э. Грей, Г. Б. Мэтьюз, Функиии Бесселя и их приложения к физике и механике, ИЛ, M., 1953.

[8] Э.А. Аринштейн, "Молекулярная теория поверхностного натяжения простых жидкостей”, Проблемы статистической физики. Труды Тюменского государственного университета. Bыn. 2, ред. Э.А. Аринштейн, Изд-во Тюменского ГУ, Тюмень, 1979, 73-87.

[9] Ф. М. Куни, А. И. Русанов, "Микроскопическая теория дисперсионных взаимодействий в капиллярных системах", Современная теория капиллярности (к 100-летию теории капиллярности Гиббса), ред. А.И. Русанов, Ф. Ч. Гудрич, Химия, Ленинград, 1980, $162-213$.

[10] П. Эгельстаф, Дж. Ринг, “Экспериментальные данные в критической области”, Физика простых жидкостей, m. 2, ред. Г. Темперли, Дж. Роулинсон, Дж. Рашбрук, Мир, М., 1973, 231-274.

Поступила в редакцию 30.06 .2005 , после доработки 19.12.2005 